\title{
Determination of Cardiac Ejection Fraction by Electrical Impedance Tomography
}

\author{
Franciane C. Peters ${ }^{1}$, Luis Paulo da S. Barra ${ }^{2}$ and Rodrigo W. dos Santos ${ }^{2}$ \\ ${ }^{1}$ Federal University of Rio de Janeiro \\ ${ }^{2}$ Federal University of Juiz de Fora \\ Brazil
}

\section{Introduction}

Cardiac ejection fraction is a clinical parameter that infers the efficiency of the heart as a pump. The ejection fraction of left ventricle (EFLV) and right ventricle (EFRV) are determined separately. However, the clinical use of EFLV is more common and it is frequently called ejection fraction (EF). By definition, the ejection fraction is calculated in the following way:

$$
E F=\frac{P V}{E D V}=\frac{E D V-E S V}{E D V}
$$

where $P V$ is the volume of blood pumped, that is given by the difference between the end-diastolic volume $(E D V)$ and the end-systolic volume $(E S V)$. Cardiac ejection fraction is a relevant parameter because it is highly correlated to the functional status of the heart. To determine the EF, different non-invasive techniques can be used, like echocardiography, cardiac magnetic resonance and computed tomography. However, they are not suitable for continuous monitoring. In this work, we numerically evaluate a new method for the continuous estimation of cardiac ejection fraction based on Electrical Impedance Tomography (EIT).

EIT is a technique that reconstructs an image of the electrical resistivity inside a domain based on protocols of current injection and potential measurement on the external boundary of the domain. Detailed description about the development of this technique can be found, for instance, in Cheney et al. (1999) and Holder (2005). The EIT has a large utilization on geophysics and monitoring of industrial activities (Kim et al., 2004; Park et al., 2008), as non-destructive technique to evaluate structures (Karhunen et al., 2009; Peters et al., 2010) and on biomedical engineering (Barber \& Brown, 1984; Brown et al., 1985; Mello et al., 2008; Trigo et al., 2004). In this last area, the EIT has been developed to detect breast and other kinds of cancer (Choi et al., 2007; Seo et al., 2004) and to monitor lung ventilation (Adler et al., 1997; Lima et al., 2007), brain activity (Polydorides et al., 2002), heart activity (Peters et al., 2009; Zlochiver et al., 2006), among others.

The special interest of the biomedical engineering in the development of the EIT is due to its safety for monitoring long periods, since it is not based on ionizing radiation. On the other hand, the EIT spacial resolution is not as high as the traditional imaging methods. 
Nevertheless, its portability, low cost and time resolution are the main advantages of the technique.

Mathematically, the EIT is classified as a non-linear inverse problem. Inverse because one wants to find the electrical resistivity of the body using measures of electrical potential on the boundary excited by known electrical current. The forward (or direct) problem related to the inverse one is to compute the electrical potential with known body resistivity and injected current. The inverse problem is non-linear, what means that there is not a linear relation between electrical resistivity and the electrical potential on the boundary. So, in general, the solution process starts with an estimated resistivity distribution and such estimative is iteratively adjusted in order to achieve an acceptable approximation for the actual resistivity distribution.

Furthermore, the inverse problem is ill-posed and ill-conditioned. In general, the number of parameters needed to represent the resistivity distribution is greater than the number of potential measures. So, in order to treat the ill-posedness of the problem, some strategies of regularization should be implemented. For instance, via the inclusion of previously known information about the resistivity distribution in the solution of the inverse problem. The problem is considered ill-conditioned because small perturbations in the measures can cause a large change in the found resistivity distribution. So, the process of image generation by EIT is very sensible to noise in the potential measures.

It is possible to see that many aspects are involved in the solution of the EIT problem and some of them were discussed in previous works of the same authors. Barra et al. (2006a) and Barra et al. (2006b) treat some computational aspects of the solution process. Peters \& Barra (2009) treats the special kind of regularization also adopted here. Peters \& Barra (2010) compares different measurement protocols and addresses the sensitivity of the process in the presence of noisy measures. Peters et al. (2009) presents the viability analysis of a biomedical application of the EIT. So the aim of this work is to revisit this particular biomedical application, describing all the procedures involved in the generation of a computational model based on cardiac magnetic ressonance images that allows the determination of the cardiac ejection fraction by the EIT. Preliminary results are presented and the potentialities and limitations of the proposed technique are discussed. The results suggest the proposed technique is a promising diagnostic tool that offers continuous and non-invasive estimation of cardiac ejection fraction.

\section{Methods}

Usual strategies to generate the EIT image is based on the discretization of the body in small parts (Borsic et al., 2001). Each part has an unknown parameter, its resistivity. So, to get a good image resolution, a great number of parameters is needed and the problem becomes ill-posed. In this strategy, regularization techniques are applied and after solving the inverse problem, the image obtained is modified by a limiarization process in order to get the final image.

In this work we adopted a different strategy to generate the EIT images. In order to monitor the cardiac ejection fraction, we assume that recent magnetic resonance images of the patient are available. So, this previous information allows the use of a different kind of parameterization in which the number of parameters is greatly reduced. 
In this section, all the methods used to solve the EIT problem will be described. First, the parameterization based on magnetic resonance images will be explained, as well as the resistivity model of the thorax. Second, the governing equations of the forward problem will be introduced in addition to the numerical methods used to solve it. Third, the inverse problem will be formulated as a minimization problem and the adopted optimization method will be presented. Finally, the computational experiments will be reported.

\subsection{Two-dimensional models based on magnetic resonance images}

\subsubsection{Parameterization}

From magnetic resonance (MR) images, the regions of interest, in this case the two ventricles, were manually segmented in two different phases: end of the systole and the end of diastole. Each curve of the segmentation was parameterized by a spline, with a minimum number of points. The left ventricle spline has 7 control points and the right one 8 points. The external boundary of the thorax and the boundaries of the lungs were also segmented. For simplicity, the shape and size of the thorax and the lungs are assumed constant during the heart cycle. Figure 1 shows a segmentation example.

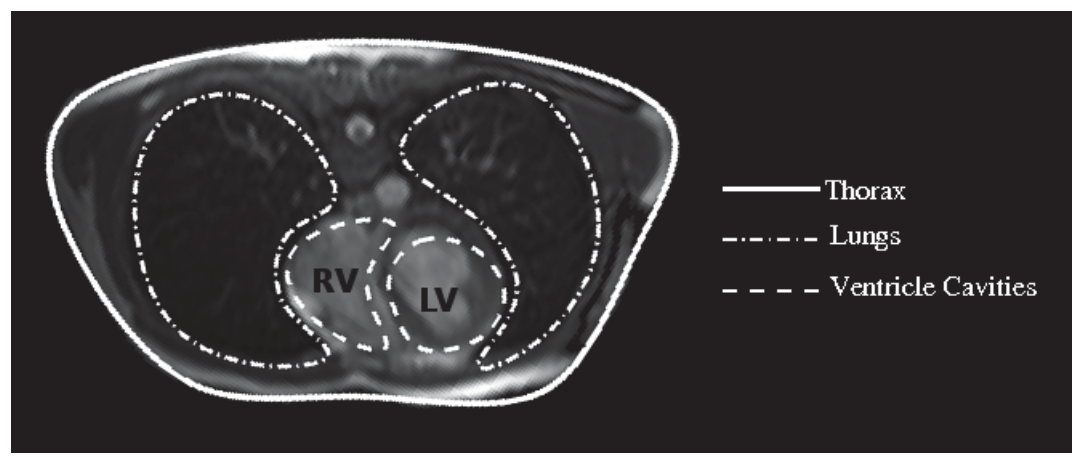

Fig. 1. Manual segmentation of an MR image. The boundaries of the lungs, ventricle cavities and thorax are represented by splines. LV and RV denotes left ventricle and right ventricle, respectively.

The goal of our method is to recover the shape of the internal cavities of the heart, presently considered in two dimensions, from electric potential measures. Therefore, with two coordinates for each control point of the spline, the methods would need to estimate a total of $30((7+8) \times 2)$ parameters. To reduce the number of parameters in half, another parameterization is used. In this, only one parameter defines the position of each control point.

During MRI segmentation we have used the same number of control points for the splines in both systolic and diastolic phase. This allows us to restrict the search space forcing that each control point $i$ belongs to a line that connects its position at systole and diastole, as shown in Fig. 2.

A linear interpolation, parameterized by a scalar $t_{i}$, is used between the values of the coordinates of each control point $i$. The spline relative to the end of systole can be obtained 


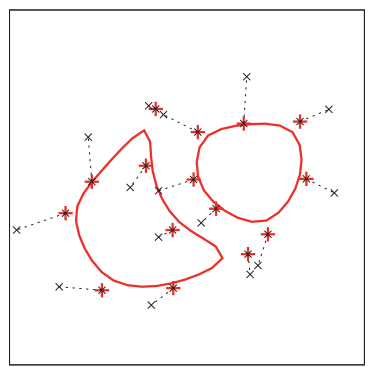

(a) Systole

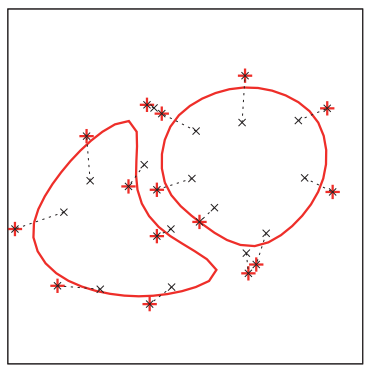

(b) Diastole

Fig. 2. The control points are represented by red crosses. The dashed lines are the search space.

with $t_{i}=0$ for all $i$, and the one relative to the end of diastole with $t_{i}=1$ for all $i$. Doing so, the method goal is to recover the shape of the ventricular cavities via the estimation of the 15 parameters $t_{i}$, with $i=1,2, \ldots, 15$.

\subsubsection{Splines}

Splines are mathematical models that associate a curve with a set of points named control points. Here we use a special type of spline called Extended Cross-Splines or Extended X-Splines (Blanc \& Schlick, 1995), for short. In this model, each control point $i$ has the coordinates $\left(x_{i}, y_{i}\right)$ and an additional parameter $s_{i} \in[0,1]$ that allows the curve $C(t)$ approximates $\left(0<s_{i} \leq 1\right)$ or sharp interpolates $\left(s_{i}=0\right)$ the control point. This feature is very interesting because it allows the definition of smooth or sharp curves. In this work, just smooth curves are represented.

Considering an Extended X-Spline model in which each segment depends on four control points, a segment $C(t)$ on the parameter range $\left(t_{k+1}, t_{k+2}\right)$ is defined as follows:

$$
\mathbf{C}(t)=F_{0} \mathbf{P}_{k}+F_{1} \mathbf{P}_{k+1}+F_{2} \mathbf{P}_{k+2}+F_{3} \mathbf{P}_{k+3},
$$

where the blending functions $F_{k}$ are obtained by the normalization of the blending functions $A_{k}(t)$ as follows:

$$
F_{k}(t)=\frac{A_{k}(t)}{A_{0}(t)+A_{1}(t)+A_{2}(t)+A_{3}(t)}
$$

and their non null part can be divided in two parts, $F_{k}^{-}$, defined between $T_{k}^{-}$and $T_{k}$, and $F_{k}^{+}$, defined between $T_{k}$ and $T_{k}^{+}$.

The functions $A_{k}(t)$ are obtained by the generic quintic polinomial $f_{p}(u)$, whose coefficients were determined in order to satisfy the constraints of the model, resulting the following expression:

$$
f_{p}(u)=u^{3}\left(10-p+(2 p-15) u+(6-p) u^{2}\right) .
$$

Hence, for the non null parts of the functions $A_{k}(t)$, we have:

$$
A_{0}(t)=f_{p_{k-1}}\left(u_{0}\right), A_{1}(t)=f_{p_{k}}\left(u_{1}\right), A_{2}(t)=f_{p_{k+1}}\left(u_{2}\right), A_{3}(t)=f_{p_{k+2}}\left(u_{3}\right)
$$


with

$$
u_{0}=\frac{t-T_{k}^{+}}{t_{k}-T_{k}^{+}}, u_{1}=\frac{t-T_{k+1}^{+}}{t_{k+1}-T_{k+1}^{+}}, u_{2}=\frac{t-T_{k+2}^{-}}{t_{k+2}-T_{k+2}^{-}}, u_{3}=\frac{t-T_{k+3}^{-}}{t_{k+3}-T_{k+3}^{-}}
$$

and

$$
\begin{gathered}
p_{k-1}=2\left(t_{k}-T_{k}^{+}\right)^{2}, p_{k}=2\left(t_{k+1}-T_{k+1}^{+}\right)^{2}, \\
p_{k+1}=2\left(t_{k+2}-T_{k+2}^{-}\right)^{2}, p_{k+2}=2\left(t_{k+3}-T_{k+3}^{-}\right)^{2} .
\end{gathered}
$$

The flexibility of the model is improved by the introduction of new degree of freedom $s_{k}$. In each control point $\mathbf{P}_{k}$ this new parameter affects the values $T_{k-1}^{+}$e $T_{k+1}^{-}$as follows:

$$
T_{k-1}^{+}=t_{k}+s_{k}, T_{k+1}^{-}=t_{k}-s_{k}
$$

In other words, one can say that the parameter $s$ controls the distance between the curve and the control point, what allows the approximation or the interpolation of these points, as shown in Fig. 3.

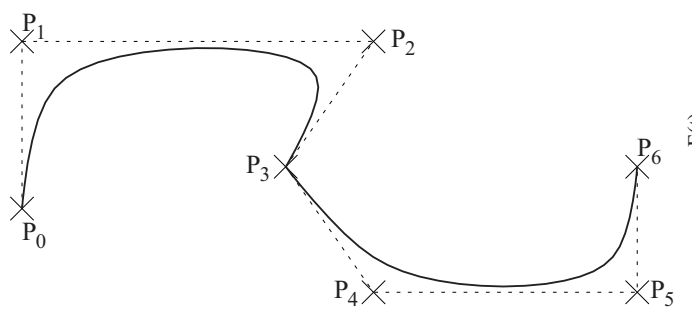

(a) Shape of the curve

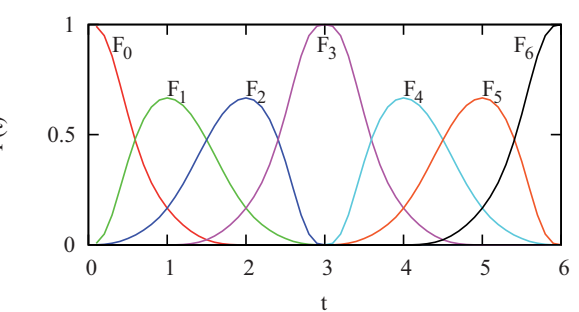

(b) Blending functions

Fig. 3. The values of the parameters of each control point are: $s_{0}=0, s_{1}=1, s_{2}=0, s_{3}=0$, $s_{4}=0, s_{5}=1, s_{6}=0$. These figures were inspired by the work of Blanc \& Schlick (1995).

Although the expressions used to implement the Extended X-spline were rewritten above, detailed description about this and other types of splines can be found in Blanc \& Schlick (1995).

\subsubsection{Resistivity model}

The proposed 2D model splits the domain in regions that represent different biological tissues, heart cavities, lungs and torso, each one associated with a different electrical resistivity.

Grimnes (2008) presents the main factors that influence the properties of biological tissues. Although they may be classified in only four groups ( epithelium, muscle, connective tissue and nervous tissue), the tissues can be divided in thirty kinds in accordance to their electrical properties (Gabriel, 1996). In addition, the value of the resistivity of each tissue depends on the frequency of the electrical current, on the temperature, on the presence of water, among other issues.

In this work, we assume the resistivity of each tissue as known, constant and isotropic. These are all simplified assumptions, since biological tissues are usually heterogeneous and 
anisotropic. However, biological tissues are difficult to characterize, and the reported values vary substantially in the literature. Table 1 presents some resistivity values for blood, heart and lung found in the literature.

\begin{tabular}{|c|c|}
\hline Tissue & Resistivity $(\Omega \mathrm{cm})$ \\
\hline \multirow{3}{*}{ Blood } & 150 (Barber \& Brown, 1984) \\
& 150 (Yang \& Patterson, 2007) \\
& 100 (Schwan \& Kay, 1956) \\
\hline \multirow{2}{*}{ Heart } & 400 (Patterson \& Zhang, 2003) \\
& 250 (Yang \& Patterson, 2007) \\
& $400-800$ (Baysal \& Eyuboglu, 2000) \\
\hline \multirow{2}{*}{ Lung } & $727-2363$ (Barber \& Brown, 1984) \\
& 1400 (Patterson \& Zhang, 2003) \\
& $600-2000$ (Baysal \& Eyuboglu, 2000) \\
\hline
\end{tabular}

Table 1. Resistivity values of biological tissues that are found in the literature.

For the remaining tissues that compose the section of the thorax, called here torso region, Bruder et al. (1994) proposes a mean resistivity of $500 \Omega \mathrm{cm}$. The resistivity of the air is $10^{20} \Omega \mathrm{cm}$, but the resistivity of the lung filled of air is difficult to determine. Rush et al. (1963) presents a very simplified resistivity distribution model of the thorax characterized by the presence of cavities filled of blood, surrounded by homogeneous material with resistivity ten times greater. The same scheme, properly extended to include the lung regions, is used in this work. Preliminarily, the resistivity of the blood is here taken as $100 \Omega \mathrm{cm}$ and the torso to be $1000 \Omega \mathrm{cm}$. Two different values were tested for the resistivity of the lungs: $20000 \Omega \mathrm{cm}$ (Ratio of Lung to Torso resistivity, RLT $=20)$ and $50000 \Omega \mathrm{cm}(\mathrm{RLT}=50)$.

\subsection{Forward problem}

The forward problem consists on calculating the electrical potential on the external boundary of the torso that is generated by the current injection on a pair of electrodes. Figure 4 presents the simplified model of the thorax.

Given that our 2D model has three regions with different but constant and isotropic conductivities (heart cavities full of blood, $\Omega_{B}$, lungs, $\Omega_{L}$, and torso, $\Omega_{T}$ ) the electrical potential $u$ at each point of the regions must satisfy Laplaces' equation:

$$
\nabla^{2} u(\mathbf{x})=0, \quad \mathbf{x} \in \Omega,
$$

the following boundary conditions:

$$
\begin{gathered}
\frac{1}{\rho_{T}} \frac{\partial u}{\partial \mathbf{n}}=J_{i}, \quad \mathbf{x} \in \Gamma_{3}^{i e} \\
\frac{\partial u}{\partial \mathbf{n}}=0, \quad \mathbf{x} \in\left(\Gamma_{3}-\Gamma_{3}^{i e}\right)
\end{gathered}
$$


and these compatibility equations:

$$
\begin{array}{ll}
\rho_{L} \nabla u=\rho_{T} \nabla u, & \mathbf{x} \in \Gamma_{1} \\
\rho_{B} \nabla u=\rho_{T} \nabla u, & \mathbf{x} \in \Gamma_{2}
\end{array}
$$

where $\Omega=\Omega_{L}+\Omega_{B}+\Omega_{T}, \Gamma_{1}$ is the interface between the lung and torso region, $\Gamma_{2}$ is the interface between the blood and the torso region, $\Gamma_{3}$ is the external boundary of the thorax, $\Gamma_{3}^{i e}$ is the part of $\Gamma_{3}$ where the ith electrode is, $J_{i}$ is the electrical current injected on the i-th electrode and $\rho_{L}, \rho_{B}$ and $\rho_{T}$ are the resistivities of the lung, blood and torso, respectively.

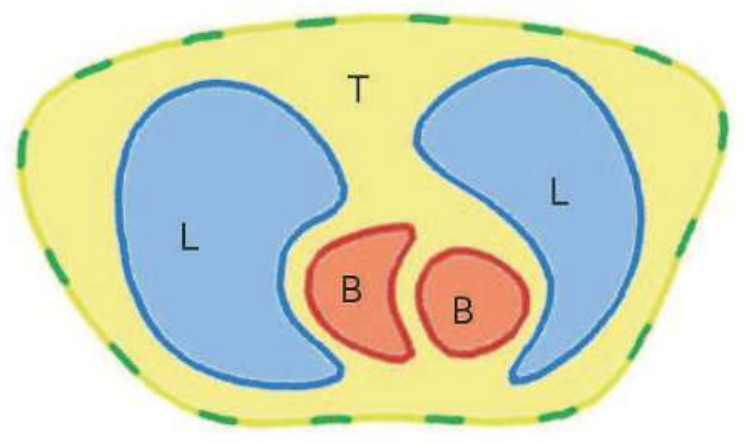

Fig. 4. The simplified thorax model. Here, the electrodes are represented in green. The regions $\mathbf{L}$ represent the lungs, $\mathbf{B}$ the blood and $\mathbf{T}$ the torso.

\subsubsection{Numerical solution of Laplace's equation}

In order to solve Equation 10 for each subregion the Boundary Element Method (BEM) is used. Further details about this technique can be found in Brebbia et al. (1984). The integral equation of BEM for this problem is

$$
c(\xi) u(\xi)+\int_{\Gamma} p^{*}(\xi ; \mathbf{x}) u(\xi ; \mathbf{x}) d \Gamma(\mathbf{x})=\int_{\Gamma} u^{*}(\xi ; \mathbf{x}) p(\xi ; \mathbf{x}) d \Gamma(\mathbf{x}),
$$

where $\xi$ is the collocation point, $\Gamma$ is the boundary of the sub-domain, $u$ is the electrical potential, $p$ is its derivative, $u^{*}$ and $p^{*}$ are the fundamental solutions for the potential and its normal derivative, respectively, and $c(\xi)$ is a function of the boundary shape, whose value is 0 if $\xi$ is outside of the domain, 1 if $\xi \in \Omega$ and $\beta / 2 \pi$ if $\xi \in \Gamma$. The parameter $\beta$ is the angle between the left and right tangents at the collocation point $\xi$.

To obtain a numerical solution for Equation 15, the boundary of the body is discretized. The external boundary is divided in $N_{0}$ elements and each subregion boundary in $N_{k}$ elements. In this work, the element adopted approximates the geometry linearly and the value of the electrical potential is considered constant in each element. In this case, the parameter $\beta=\pi$ and then $c(\xi)=0.5$ if $\xi \in \Gamma$. Each boundary element has two nodes for the geometrical definition and a centered node, called functional node, where the potential and 
its derivative are computed. Thus, the discretized form of Equation 15 for each subregion $k$ allows evaluating the potential at each functional node as follows

$$
c\left(\xi_{i}\right) u\left(\xi_{i}\right)+\sum_{j=1}^{N_{k}} u_{j} \int_{\Gamma_{j}} p^{*} d \Gamma_{J}=\sum_{j=1}^{N_{k}} p_{j} \int_{\Gamma_{j}} u^{*} d \Gamma_{J},
$$

where $u_{j}$ and $p_{j}$ represent the potential and its normal derivative at the $j$-th functional node. The regular integrals are computed numerically by the usual Gauss Quadrature scheme and the singular ones are computed analytically.

The application of Equation 16 for each sub-domain $\Omega_{k}$, in addition to the boundary and compatibility conditions (Equations 11 to 14) for the potential and its normal derivative at the functional nodes of the interface elements at $\Gamma_{0 k}$, yields a linear system of algebraic equations that can be expressed in the matrix form as follows:

$$
\mathrm{Hu}=\mathrm{Gp},
$$

where $\mathbf{u}$ and $\mathbf{p}$ are vectors that store the values of potential and its derivative, respectively, at the functional nodes of the boundary elements. The matrices $\mathbf{H}$ and $\mathbf{G}$ store the respective computed coefficients.

The number of unknowns is the number of the external boundary elements, in which the potential or the flux is unknown, in addition to the double of interface elements, in which the potential and the flux are unknowns. After collect all of them at the vector $\mathbf{y}$, Equation 17 can be rewritten as

$$
\mathrm{Ay}=\mathbf{b},
$$

where $\mathbf{A}$ is the matrix of coefficients and $\mathbf{b}$ is the free vector of the linear system.

After determining the unknowns at the boundary, the values of the electrical potential at the nodes in the center of the electrodes without prescribed values are collected in the vector $\mathbf{V}$. Such vector will be compared with the vector of measures $\overline{\mathbf{V}}$ during the process of solving the inverse problem.

The implementation of the Boundary Elements Method to solve Laplace's equation was written in Fortran language.

\subsection{The inverse problem}

As was said before, the aim of the EIT is to generate an image of the electrical resistivity from measures of electrical potential at the external boundary. This problem can be formulated as a minimization problem in which one wants to find the model of electrical resistivity that minimizes the distance between measured $(\overline{\mathbf{V}})$ and computed $(\mathbf{V})$ potentials. In this work, the objective is to recover the shape of the ventricular cavities under the circumstances explained before. Therefore, the resistivity model is obtained via the estimation of the vector $\mathbf{t}$ defined in Section 2.1.1. In this case, the vector contains 15 parameters $t_{i}$, with $i=1,2, \ldots, 15$ as described 
before. In other words, the goal is to estimate the parameter vector $\mathbf{t}$ that minimizes $f$ :

$$
f=\frac{1}{2} \mathbf{R}(\mathbf{t})^{T} \mathbf{R}(\mathbf{t})
$$

with

$$
\mathbf{R}(\mathbf{t})=\overline{\mathbf{V}}-\mathbf{V}(\mathbf{t})
$$

where $f$ is the objective function $\left(f: \mathbb{R}^{n} \rightarrow \mathbb{R}\right), \mathbf{R}(\mathbf{t})$ is the residual function $\left(\mathbf{R}: \mathbb{R}^{n} \rightarrow \mathbb{R}^{m}\right)$, $m$ is the number of measures and $n$ is the number of parameters. The number of measures depends on the adopted protocol to inject current and measure electrical potential.

Equation 19 shows that the problem leads to a non-linear least square problem. So, many techniques can be used to solve it. The so called global convergent methods, for example, Genetic Algorithms (Eiben \& Smith, 2003; Michalewicz, 1996), has the advantage of avoid the convergence to local minimum. However, they demand a large number of evaluations of the objective function. On the other hand, the local minimization methods converge faster to the minimum because they use the derivatives of the objective function with respect to the parameters. They also demand a suitable initial approximation to converge to the global minimum. Hybrid strategies, that combine the advantages of local and global methods can be used with success (Hsiao et al., 2001; Peters et al., 2010). In this work, the features of the problem allows the use of a local strategy, the called Levenberg-Marquard Method, that will be briefly described as follows.

\subsubsection{Levenberg-Marquardt method}

The Levenberg-Marquardt Method was adopted to solve the non-linear least square problem represented by Equation 19. The detailed description about this method is vastly found in the literature (Dennis \& Schnabel, 1996; Fletcher, 1980; Madsen et al., 2004). The Levenberg-Marquardt Method can be understood as the Gauss-Newton method modified by the model trust region approach. In this method, the minimizer of the non-linear least-square problem is obtained iteratively. Each update of $\mathbf{t}$ is given by the minimizer $\mathbf{t}_{+}$of the following constrained linear least-square problem:

$$
\begin{gathered}
\text { minimizes }\left\|\mathbf{R}\left(\mathbf{t}_{0}\right)+\mathbf{J}\left(\mathbf{t}_{0}\right)\left(\mathbf{t}_{+}-\mathbf{t}_{0}\right)\right\|_{2} \\
\text { subject to }\left\|\mathbf{t}_{+}-\mathbf{t}_{0}\right\|_{2} \leq \delta_{0} .
\end{gathered}
$$

where $\mathbf{t}_{0}$ is the current value for the vector of minimization parameters and $\mathbf{t}_{+}$is the updated vector. $\mathbf{R}$ is the same residual function mentioned before. $\mathbf{J} \in \mathbb{R}^{m \times n}$ is the Jacobian matrix, storing the derivatives of each element of the residual vector with respect to the optimization variables $\left(J_{i j}=\partial R_{i} / \partial t_{j}\right)$. $\delta_{0}$ is the initial value of the radius of the trust region.

The solution of this constrained minimization problem is the updated vector of variables $\mathbf{t}_{+}$:

$$
\mathbf{t}_{+}=\mathbf{t}_{0}-\left(\mathbf{J}\left(\mathbf{t}_{0}\right)^{T} \mathbf{J}\left(\mathbf{t}_{0}\right)+\mu \mathbf{I}\right)^{-1} \mathbf{J}\left(\mathbf{t}_{0}\right)^{T} \mathbf{R}\left(\mathbf{t}_{0}\right)
$$

where $\mathbf{I}$ is the identity matrix and $\mu$ is the parameter that modifies the Gauss-Newton method. If $\left\|\left(\mathbf{J}\left(\mathbf{t}_{0}\right)^{T} \mathbf{J}\left(\mathbf{t}_{0}\right)\right)^{-1} \mathbf{J}\left(\mathbf{t}_{0}\right)^{T} \mathbf{R}\left(\mathbf{t}_{0}\right)\right\|_{2} \leq \delta_{0}, \mu=0$, otherwise, $\mu \neq 0$. 
There are some different implementations of this method with respect to the update of the parameter $\mu$. In this work, the implementation of the Levenberg-Marquardt Method is provided by MINPACK-1, a standard package of subroutines written in Fortran language to solve non-linear equations and non-linear least squares problems, that is available at the Netlib repository (http://www.netlib.org/minpack). More details about the adopted implementation can be found in Moré et al. (1980). In the numerical experiments presented in this work, the subroutine LMDIF of MINPACK-1 was used. Such subroutine demands only the computation of the residual function $\mathbf{R}(\mathbf{t})$. The jacobian matrix is approximated by finite differences. So, the element $J_{i j}$ of the Jacobian matrix is computed as follows:

$$
J_{i j}=\frac{\partial R_{i}}{\partial t_{j}} \approx \frac{R_{i}\left(\mathbf{t}+h_{j} \mathbf{e}_{j}\right)-R_{i}(\mathbf{t})}{h_{j}}
$$

where $h_{j}$ is a small finite perturbation at the $j$-th element of the original vector of optimization variables $\mathbf{t}$ and $\mathbf{e}_{j}$ is the $j$-th column of the identity matrix.

The value of the parameter $h_{j}$ is computed by the product $\sqrt{\varepsilon} t_{j}$, where $\varepsilon$ is a parameter provided by the user. If the machine precision is greater than the computed $h_{j}$, this value is substituted by the machine precision.

\subsection{Numerical experiments}

\subsubsection{Stimulation patterns}

An important aspect of the EIT problem is the choice of the protocols of current injection and electrical potential measurements. Since the problem is ill-conditioned, the suitable choice can be determinant to the success of the image generation. However, a deep study of the influence of different protocols on the solution of the inverse problem is not the focus of this work. More information on this topic can be found in other works, for instance, Peters \& Barra (2010). Here we are limited to compare two patterns of electrical current injection. The first is called diametrical and the second is called alternative. Furthermore, all the experiments were done considering 16 electrodes equally spaced on the external boundary of the torso.

The name of the first pattern, diametrical, comes from an analogy. If the domain were circular, the electrodes used to inject current are diametrically opposed. Although the torso is not circular, the name of the pattern remains. In this pattern, 8 different cases of current injection is taken and 13 measures of potential for each case. So, the diametrical pattern yields 104 measures.

The second pattern, here called alternative, is a tentative of illuminating the region of interest better than other regions. Therefore, in this pattern, the electrodes used to inject current, the driven electrodes, are taken near to the heart. So, 6 cases of current injection with 13 measures each one give a total of 78 measures. Each double arrow of Fig. 5 indicates the driven electrode pair in each case of current injection. In both patterns, measurements on driven electrodes are not considered.

It is important to note that, in this work, the "measured" electrical potential values $(\overline{\mathbf{V}})$ were also synthetically generated, i.e., also numerically obtained. 


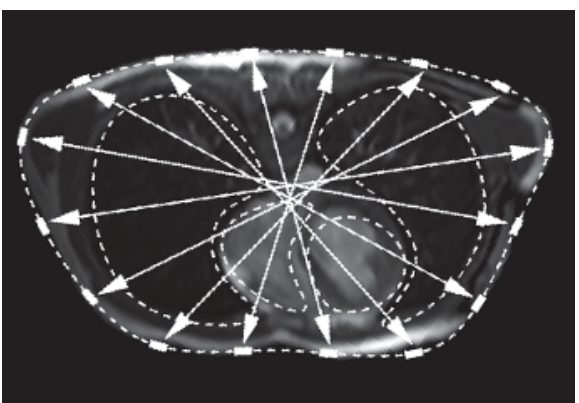

(a) Diametrical Pattern

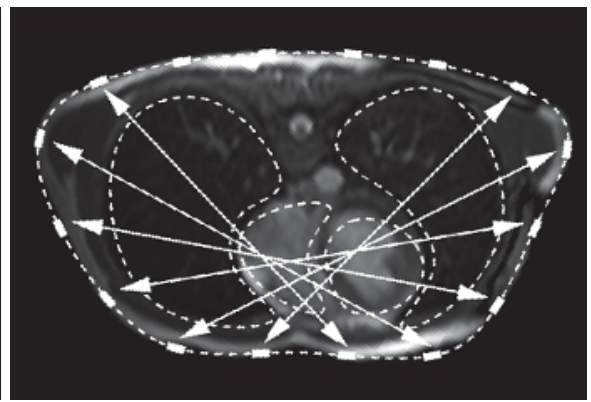

(b) Alternative Pattern

Fig. 5. Two stimulation patterns used in this work. Each double arrow indicates one pair of driven electrodes.

\subsubsection{Problem}

From MR images taken at the end of the systole and at the end of the diastole the cardiac ventricles were manually segmented. In this work we are considering an image of just one transversal section of the thorax. Therefore, the problem is treated as $2 \mathrm{D}$ and an approximation of the ejection fraction is needed.

Here, the section of the cavities were assumed to be proportional to their volumes, i.e. a cylindrical approximation. So that, in accordance to Equation 1, EF is approximated by:

$$
E F=\frac{E D A-E S A}{E D A}
$$

where EDA is the end-diastolic area and ESA is the end-systolic area.

Therefore, after segmentation, the EF is calculated in accordance to Equation 25. The EF of the left ventricle is $59.24 \%$ and the EF of the right ventricle is $29,95 \%$. These values characterize the initial situation of the heart cicle. From this moment, the EIT can be used to monitor the EF.

Later, a cardiac dysfunction was synthetically generated. In this simulated dysfunction model the end-diastolic volume is the same as in the normal cycle but the end-systolic volume is greater than the normal one. In this pathological situation new cardiac cycle, the EF of the left ventricle is $33.01 \%$ and the $\mathrm{EF}$ of the right ventricle is $16.19 \%$. These dysfunction values are the target values to be estimated by the here proposed method.

As mentioned in Section 2.1, we have also tested the methods considering two different 2D models. Each with a different value for the resistivity of the lungs: $20000 \Omega \mathrm{cm}(\mathrm{RLT}=20)$ and $50000 \Omega c m($ RLT $=50)$.

As was said before, the solution depends on the initial guess provided for the local minimization method. So that, for each of 4 optimization problems ( 2 stimulus patterns times 2 RLT models) we have tested the optimization method with two different initial guesses. One guess corresponds to the shape of the ventricles at the end of the diastole of normal heart, i.e. $t_{i}=1, \forall i$ and the other at the end of the systole for the normal tissue, i.e. $t_{i}=0, \forall i$. The initial 
guesses and the target can be compared in Fig. 6. Thus, the method was executed a total of 8 times ( 2 stimulus patterns times 2 RLT models times 2 initial guesses). Each execution computes the parameters $\mathbf{t}$ of the end of the systole. The areas inside the curves defined by $\mathbf{t}$ are the ESA. These values together with the known EDA values, that is supposed to be the same as the initial condition of the heart cycle, are used with Equation 25 to compute the EF. Therefore, each execution yields 2 values: EF of the left and right ventricle.

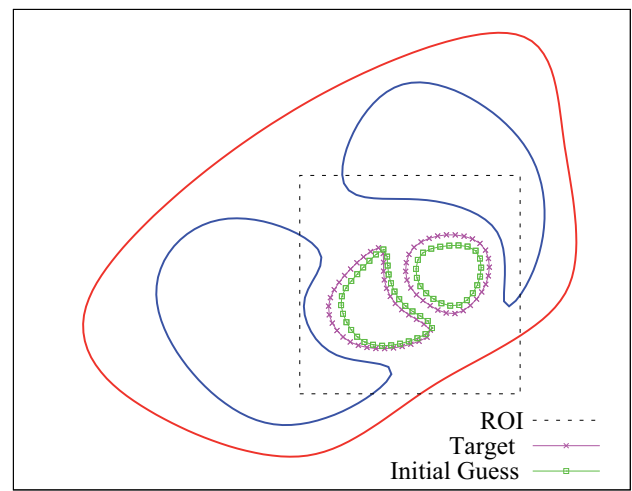

(a) $t_{i}=0, \forall i$

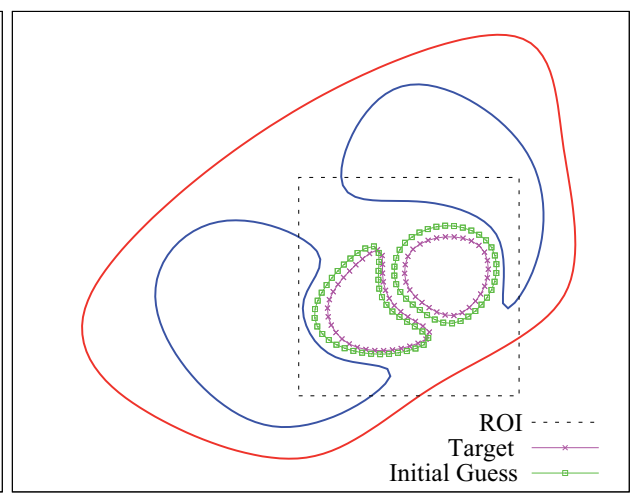

(b) $t_{i}=1, \forall i$

Fig. 6. A typical target (pink) and the two initial guesses (green) given to the optimization procedure. The first one corresponds to the systole and the second one to the diastole. ROI denotes the region of interest.

\section{Results}

Table 2 presents the results of our numerical experiments that aim the EF estimation of the synthetically generated cardiac dysfunction. The columns present the results for the models with different values for the resistivity of the lungs: $20000 \Omega \mathrm{cm}(\mathrm{RLT}=20)$ and $50000 \Omega \mathrm{cm}$ $($ RLT $=50)$. Each couple of rows presents the comparison of the two stimulation patterns implemented: diametrical and alternative. In addition, for each pair (stimulus pattern, RLT) results are presented for two different initial guesses. The first one corresponds to the shape of the ventricles at the end of the systole for the normal heart, i.e. $t_{i}=0, \forall i$ and the other at the end of the diastole of the normal heart, i.e. $t_{i}=1, \forall i$. The last row of the table presents the target values for comparison: $16.19 \%$ the EF of right ventricle (RV) and $33.01 \%$ the EF of left ventricle (LV).

Table 3 shows the relative errors between the values of EF obtained in each execution and the target values for each ventricle. These values are computed as

$$
\Delta \%=100 \times \frac{|\tilde{E F}-E F|}{E F},
$$

where $\Delta \%$ is the error, $\tilde{E F}$ is the value achieved by the inverse problem solution for the ejection fraction and $E F$ is the target value. The relative errors are used to compute the mean relative errors used to compare patterns, initial guesses and models. 


\begin{tabular}{|lcccc|}
\hline \multicolumn{5}{|c|}{ Ejection Fraction (\%) } \\
Initial & RLT $=50$ & RLT $=20$ \\
Guess & RV & LV & RV & LV \\
\hline \multicolumn{5}{|c|}{ Diametrical Pattern } \\
$t_{i}=0$ & 13.00 & 34.41 & 15.32 & 34.22 \\
$t_{i}=1$ & 16.09 & 32.21 & 15.80 & 33.04 \\
\hline \multicolumn{5}{c}{ Alternative Pattern } \\
$t_{i}=0$ & 12.97 & 35.86 & 20.54 & 29.94 \\
$t_{i}=1$ & 18.72 & 32.84 & 20.89 & 29.40 \\
\hline Target & $\mathbf{1 6 . 1 9}$ & 33.01 & $\mathbf{1 6 . 1 9}$ & 33.01 \\
\hline
\end{tabular}

Table 2. Values of the ejection fraction estimated for the synthetic cardiac dysfunction for two resistivity models (RLT $=20$ and RLT $=50$ ), two different stimulation patterns (diametrical and alternative) and two initial guesses $\left(t_{i}=0\right.$ and $\left.t_{i}=1\right)$. The last row shows the target values of the EF.

\begin{tabular}{|ccccc|}
\hline \multicolumn{5}{|c|}{ Relative Errors (\%) } \\
Initial & RLT $=50$ & RLT $=20$ \\
Guess & RV & LV & RV & LV \\
\hline \multicolumn{5}{|c|}{ Diametrical Pattern } \\
$t_{i}=0$ & 19.70 & 4.24 & 5.37 & 3.67 \\
$t_{i}=1$ & 0.62 & 2.42 & 2.41 & 0.09 \\
\hline \multicolumn{5}{c}{ Alternative Pattern } \\
$t_{i}=0$ & 19.89 & 8.63 & 26.87 & 9.30 \\
$t_{i}=1$ & 15.63 & 0.51 & 29.03 & 10.94 \\
\hline
\end{tabular}

Table 3. Relative errors of the obtained EF with respect to the target values.

Figure 7 allows a geometrical comparison between the final results and the actual (target) curves. In order to make the visualization easier, these figures show the region of interest defined in Fig. 6 without the curves of the lungs. It is important to emphasize that, to make the comparison fair, the results presented in these figures were obtained with the same initial guess, $t_{i}=1, \forall i$.

The results show that, except in one case, the error of the ejection fraction of the left ventricle is smaller than the right ventricle value. The mean relative error of the eight results of the left ventricle results is $4.98 \%$ while the right ventricle is $14.94 \%$.

Moreover, except in one case, the diametrical pattern provides results closer to the actual values than the alternative pattern. The diametrical pattern provides a mean relative value of $4.82 \%$ while the mean error of the alternative pattern is $15.10 \%$. 


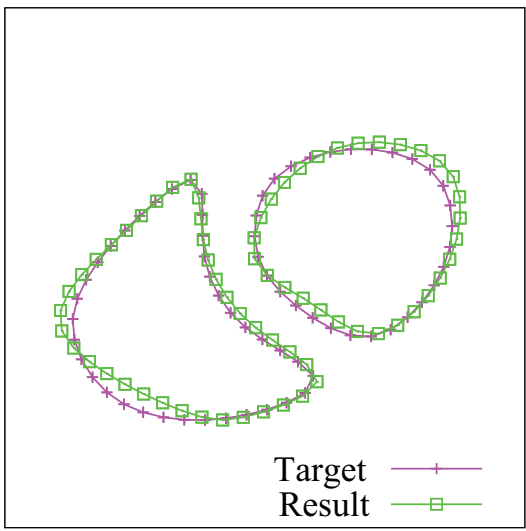

(a) $\mathrm{RLT}=50$ - Diametrical

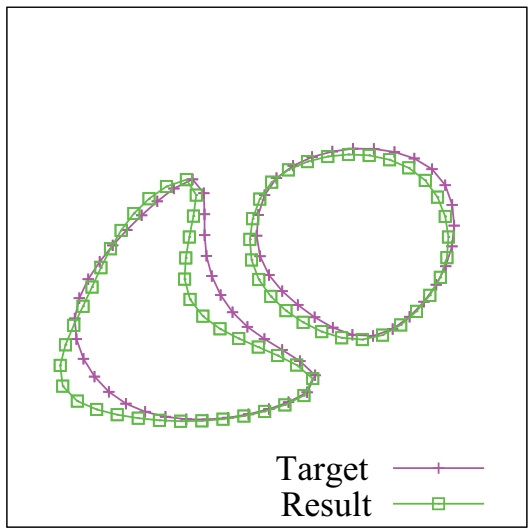

(c) RLT $=50$ - Alternative

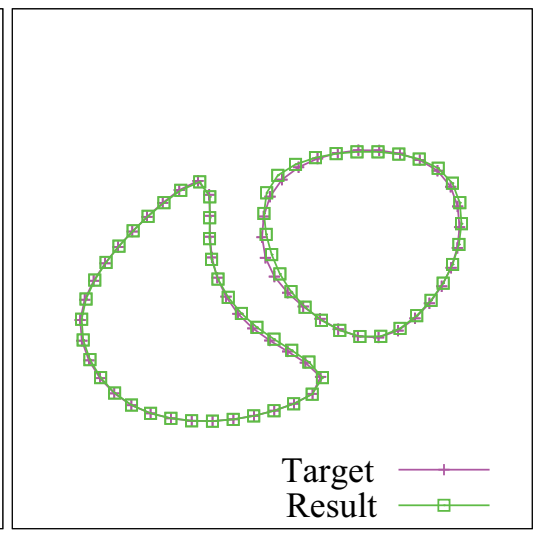

(b) RLT = 20 - Diametrical

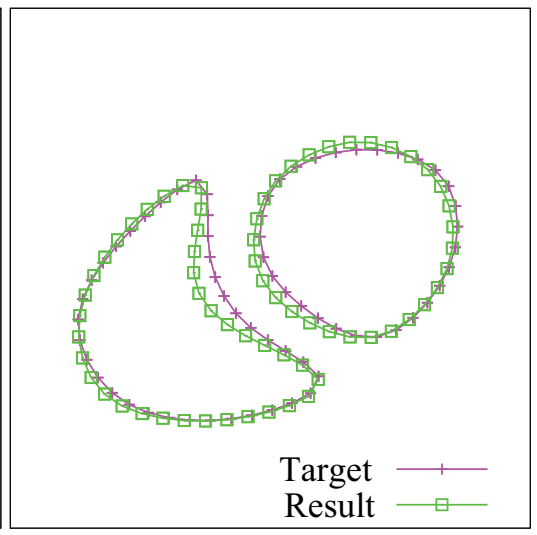

(d) RLT = 20 - Alternative

Fig. 7. Some results for the diametrical and the alternative pattern and the target.

About the initial guess, both of them provided good results. However, in this experiments, the best results were obtained with the guess of the original diastole curve, $t_{i}=1$, with a mean relative error of $7.70 \%$ against an error of $12.21 \%$ for the other initial guess.

The geometrical results presented in Fig. 7, showing only the ventricular cavities, suggest that they become worse in the case the lung resistivity is greater. This behavior is expected because greater resistivities around the region of interest tend to block the electrical current to reach this area. For instance, for the best experimented pattern, the diametrical one, the mean relative error obtained with the greatest resistivity $(\mathrm{RLT}=50)$ is $3.37 \%$ while the mean relative error obtained with the other lung resistivity $(\mathrm{RLT}=20)$ is $1.44 \%$.

The best result can be considered the one obtained for RLT $=20$, diametrical pattern and $t_{i}=1$. The relative error in the value of the ejection fraction is $0.09 \%$ for the left ventricle and $2.41 \%$ for the right ventricle. Figure $7(b)$ shows this result. In this case it is very difficult to see the difference between the result and the target. 


\section{Discussions and conclusions}

The presented results suggest that the proposed methodology allows a suitable indication of the cardiac ejection fraction. We have observed that the error in the ejection fraction predictions for the right ventricle are greater than those found for the left ventricle and this is in agreement with other techniques, such as with echocardiography.

Concerning the different patterns for current injection tested in this work, the errors obtained with the diametrical pattern are smaller than those using the alternative pattern, in general. However this fact does not discard the use of the alternative pattern, as it presents good results and spends around $19 \mathrm{~min}$. in a Pentium 4, $3.00 \mathrm{GHz}$, for a complete solution, 25\% less then the diametrical.

Comparing the results obtained with different lung resistivities we may conclude that the inverse problem becomes more difficult to be solved as the RLT increases. Therefore, the results suggest the current injection should be triggered during the expiratory phase, when the air volume and the corresponding lung resistivity are smaller.

The preliminary results presented in this work suggest the proposed technique is a promising diagnostic tool that may offer continuous and non-invasive estimation of cardiac ejection fraction. However, the use of the EIT in real applications demands further improvements. The model could be improved, for instance, by the implementation of the complete electrode model (Cheney et al., 1999).

Another point is that, in this work, we assume the resistivities of the tissue known. Future works should include the resistivities of the tissue as parameters of the inverse problem, as well as deeper studies about the electrical properties of biological tissues. Furthermore, the behavior of the proposed method when subjected to real data should be evaluated.

\section{Acknowledgements}

This work was partially supported by FAPEMIG, UFJF, CAPES, CNPq and FINEP. In particular, the first author would like to thank CAPES for the Master's scholarship at Federal University of Juiz de Fora and CNPq for the Doctoral scholarship at Federal University of Rio de Janeiro.

\section{References}

Adler, A., Amyot, R., Guardo, R., Bates, J. H. T. \& Berthiaume, Y. (1997). Monitoring changes in lung air and liquid volumes with electrical impedance tomography, Journal of Applied Physiology 83(5): 1762-1767.

Anderson, E., Bai, Z., Bischof, C., Blackford, L. S., Demmel, J., Dongarra, J. J., Du Croz, J., Hammarling, S., Greenbaum, A., McKenney, A. \& Sorensen, D. (1999). LAPACK Users' guide (third ed.), Society for Industrial and Applied Mathematics, Philadelphia, PA, USA.

Barber, D. C. \& Brown, B. H. (1984). Applied potential tomography, Journal of Physics E-Scientific Instruments 17(9): 723-733. 
Barra, L. P. S., Peters, F., Martins, C. P. \& Barbosa, H. (2006). Computational experiments in electrical impedance tomography, XXVII Iberian Latin American Congress on Computational Methods in Engineering, Belém, Brasil.

Barra, L., Santos, R., Peters, F., Santos, E. \& Barbosa, H. (2006). Parallel computational experiments in electrical impedance tomography, 18th Symposium on Computer Architecture and High Performance Computing, Vol. 1, Sociedade Brasileira de Computação, High Perfomance Computing in the Life Sciences, Ouro Preto, Brasil, pp. 7-13.

Baysal, U. \& Eyuboglu, B. M. (2000). Tissue resistivity estimation in the presence of positional and geometrical uncertainties, Physics in Medicine and Biology 45(8): 2373-2388.

Blanc, C. \& Schlick, C. (1995). X-splines: A spline model designed for the end-user, SIGGRAPH, ACM - Association for Computing Machinery, Los Angeles, EUA, pp. 377-386.

Borsic, A., McLeod, C., Lionheart, W. \& Kerrouche, N. (2001). Realistic 2d human thorax modelling for eit, Physiological Measurement 22(1): 77-83.

Brebbia, C., Telles, J. C. F. \& Wrobel, L. C. (1984). Boundary Elements Techniques: Theory and Applications in Engineering, Springer-Verlag.

Brown, B. H., Barber, D. C. \& Seagar, A. D. (1985). Applied potential tomography - possible clinical applications, Clinical Physics and Physiological Measurement 6(2): 109-121.

Bruder, H., Scholz, B. \& Abrahamfuchs, K. (1994). The influence of inhomogeneous volume conductor models on the ecg and the mcg, Physics in Medicine and Biology 39(11): 1949-1968.

Cheney, M., Isaacson, D. \& Newell, J. C. (1999). Electrical impedance tomography, SIAM Review 41(1): 85-101.

Choi, M. H., Kao, T. J., Isaacson, D., Saulnier, G. J. \& Newell, J. C. (2007). A reconstruction algorithm for breast cancer imaging with electrical impedance tomography in mammography geometry, IEEE Transactions on Biomedical Engineering 54(4): 700-710.

Dennis, J. \& Schnabel, R. B. (1996). Numerical Methods for Unconstrained Optimization and Nonlinear Equations, SIAM.

Eiben, A. E. \& Smith, J. E. (2003). Introduction to Evolutionary Computing, Springer.

Fletcher, R. (1980). Practical methods of optimization, Wiley-Interscience, New York, NY, USA.

Gabriel, C. (1996). The dielectric properties of biological tissue: I. literature survey, Phys. Med. Biol.

Grimnes, S. (2008). Bioimpedance and Bioelectricity Basics, Academic Press.

Holder, D. (2005). Electrical Impedance Tomography: Methods, History and Applications, Medical Physics 32: 2731.

Hsiao, C. T., Chahine, G. \& Gumerov, N. (2001). Application of a hybrid genetic/powell algorithm and a boundary element method to electrical impedance tomography, Journal of Computational Physics 173: 433-454.

Karhunen, K., Seppanen, A., Lehikoinen, A., Monteiro, P. J. M. \& Kaipio, J. P. (2009). Electrical resistance tomography imaging of concrete, Cement and Concrete Research 40(1): 137-145.

Kim, M., Kim, K. \& Kim, S. (2004). Phase boundary estimation in two-phase flows with electrical impedance tomography, Int. Comm. Heat Transfer 31(8): 1105-1114. 
Lima, C. R., Mello, L. A. M., Lima, R. G. \& Silva, E. C. N. (2007). Electrical impedance tomography through constrained sequential linear programming: a topology optimization approach, Measurement Science and Technology 18(9): 2847-2858.

Madsen, K., Nielsen, H. \& Tingleff, O. (2004). Methods for non-linear least squares problems.

Mello, L. A. M., de Lima, C. R., Amato, M. B. P., Lima, R. G. \& Silva, E. C. N. (2008). Three-dimensional electrical impedance tomography: A topology optimization approach, Biomedical Engineering, IEEE Transactions on 55(2): 531-540.

Michalewicz, Z. (1996). Genetic Algorithms + Data Structures = Evolution Programs, Springer Verlag.

Moré, J. J., Garbow, B. S. \& Hillstrom, K. E. (1980). User guide for MINPACK-1, Technical Report ANL-80-74, Argonne National Laboratory, Argonne, IL, USA.

Park, B. G., Moon, J. H., Lee, B. S. \& Kim, S. (2008). An electrical resistance tomography technique for the monitoring of a radioactive waste separation process, International Communications in Heat and Mass Transfer 35(10): 1307-1310.

Patterson, R. P. \& Zhang, J. (2003). Evaluation of an eit reconstruction algorithm using finite difference human thorax models as phantoms, Physiological Measurement 24(2): 467-475.

Peters, F. \& Barra, L. (2009). A strategy for parametrization refinement in the solution of a geometric inverse problem, Computational Modeling (MCSUL), 2009 Third Southern Conference on, pp. $136-142$.

Peters, F., Barra, L. \& Santos, R. (2009). Determination of cardiac ejection fraction by electrical impedance tomography - numerical experiments and viability analysis, Computational Science - ICCS 2009, Vol. 5544/2009 of Lecture Notes in Computer Science, Springer Berlin / Heidelberg, pp. 819-828.

Peters, F. C. \& Barra, L. P. S. (2010). Some numerical results on the influence of measurement strategies and load patterns in the eit inverse problem, Journal of Physics: Conference Series 224(1): 012145.

Peters, F. C., Barra, L. P. S. \& Lemonge, A. C. C. (2010). Application of a hybrid optimization method for identification of steel reinforcement in concrete by electrical impedance tomography, 2nd International Conference on Engineering Optimization.

Polydorides, N., Lionheart, W. \& McCann, H. (2002). Krylov subspace iterative thechniques: On the brain activity with electrical impedance tomography, IEEE Transactions on Medical Imaging 21(6): 596-603.

Rush, S., McFee, R. \& Abildskov, J. A. (1963). Resistivity of body tissues at low frequencies, Circulation Research 12(1): 40-50.

Schwan, H. P. \& Kay, C. F. (1956). Specific resistance of body tissues, Circulation Research 4(6): 664-670.

Seo, J., Kwon, O., Ammari, H. \& Woo, E. (2004). A mathematical model for breast cancer lesion estimation: Electrical impedance technique using ts2000 commercial system, IEEE Transactions on Biomedical Engineering 51(11): 1898-1906.

Trigo, F., Lima, R. \& Amato, M. (2004). Electrical impedance tomography using extended kalman filter, IEEE Transactions on Biomedical Engineering 51(1): 72-81.

Yang, F. \& Patterson, R. P. (2007). The contribution of the lungs to thoracic impedance measurements: a simulation study based on a high resolution finite difference model, Physiological Measurement 28(7): S153-S161. 
Zlochiver, S., Freimark, D., Arad, M., Adunsky, A. \& Abboud, S. (2006). Parametric eit for monitoring cardiac stroke volume, Vol. 27, Iop Publishing Ltd, pp. S139-S146. 


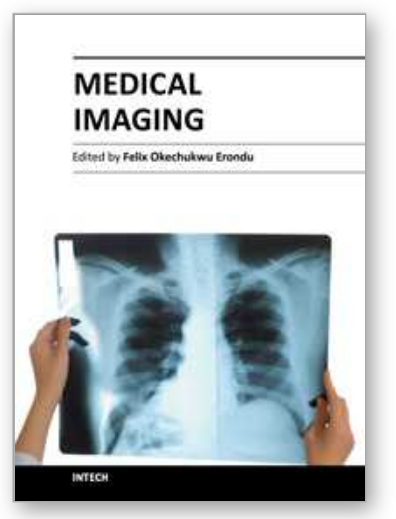

\author{
Medical Imaging \\ Edited by Dr. Okechukwu Felix Erondu
}

ISBN 978-953-307-774-1

Hard cover, 412 pages

Publisher InTech

Published online 22, December, 2011

Published in print edition December, 2011

What we know about and do with medical imaging has changed rapidly during the past decade, beginning with the basics, following with the breakthroughs, and moving on to the abstract. This book demonstrates the wider horizon that has become the mainstay of medical imaging sciences; capturing the concept of medical diagnosis, digital information management and research. It is an invaluable tool for radiologists and imaging specialists, physicists and researchers interested in various aspects of imaging.

\title{
How to reference
}

In order to correctly reference this scholarly work, feel free to copy and paste the following:

Franciane C. Peters, Luis Paulo da S. Barra and Rodrigo W. dos Santos (2011). Determination of Cardiac Ejection Fraction by Electrical Impedance Tomography, Medical Imaging, Dr. Okechukwu Felix Erondu (Ed.), ISBN: 978-953-307-774-1, InTech, Available from: http://www.intechopen.com/books/medicalimaging/determination-of-cardiac-ejection-fraction-by-electrical-impedance-tomography

\section{INTECH}

open science | open minds

\section{InTech Europe}

University Campus STeP Ri

Slavka Krautzeka 83/A

51000 Rijeka, Croatia

Phone: +385 (51) 770447

Fax: +385 (51) 686166

www.intechopen.com

\section{InTech China}

Unit 405, Office Block, Hotel Equatorial Shanghai

No.65, Yan An Road (West), Shanghai, 200040, China 中国上海市延安西路65号上海国际贵都大饭店办公楼 405 单元

Phone: +86-21-62489820

Fax: +86-21-62489821 
(C) 2011 The Author(s). Licensee IntechOpen. This is an open access article distributed under the terms of the Creative Commons Attribution 3.0 License, which permits unrestricted use, distribution, and reproduction in any medium, provided the original work is properly cited. 